ARTICLE

\title{
Interpenetrating interfaces for efficient perovskite solar cells with high operational stability and mechanical robustness
}

Qingshun Dong (1) 1,2, Chao Zhu (10 3,4, Min Chen (1) 2, Chen Jiang1', Jingya Guo1, Yulin Feng ${ }^{1}$, Zhenghong Dai², Srinivas K. Yadavalli ${ }^{2}$, Mingyu Hu (10 ${ }^{2}$, Xun Cao (1) ${ }^{4}$, Yuqian Li ${ }^{5}$, Yizhong Huang ${ }^{4}$, Zheng Liu (D) ${ }^{4}$, Yantao Shi ${ }^{1 凶}$, Liduo Wang6, Nitin P. Padture (i] ${ }^{2 \bowtie} \&$ Yuanyuan Zhou (i) ${ }^{2,7 凶}$

The perovskite solar cell has emerged rapidly in the field of photovoltaics as it combines the merits of low cost, high efficiency, and excellent mechanical flexibility for versatile applications. However, there are significant concerns regarding its operational stability and mechanical robustness. Most of the previously reported approaches to address these concerns entail separate engineering of perovskite and charge-transporting layers. Herein we present a holistic design of perovskite and charge-transporting layers by synthesizing an interpenetrating perovskite/electron-transporting-layer interface. This interface is reactionformed between a tin dioxide layer containing excess organic halide and a perovskite layer containing excess lead halide. Perovskite solar cells with such interfaces deliver efficiencies up to $22.2 \%$ and $20.1 \%$ for rigid and flexible versions, respectively. Long-term ( $1000 \mathrm{~h}$ ) operational stability is demonstrated and the flexible devices show high endurance against mechanical-bending (2500 cycles) fatigue. Mechanistic insights into the relationship between the interpenetrating interface structure and performance enhancement are provided based on comprehensive, advanced, microscopic characterizations. This study highlights interface integrity as an important factor for designing efficient, operationally-stable, and mechanically-robust solar cells.

\footnotetext{
${ }^{1}$ State Key Laboratory of Fine Chemicals, Department of Chemistry, School of Chemical Engineering, Dalian University of Technology, Dalian 116024, China.

${ }^{2}$ School of Engineering, Brown University, Providence 02912 RI, USA. ${ }^{3}$ SEU-FEI Nano-Pico Center, Key Laboratory of MEMS of Ministry of Education, Collaborative Innovation Center for Micro/Nano Fabrication, Device and System, Southeast University, Nanjing 210096 , China. ${ }^{4}$ School of Materials Science and Engineering, Nanyang Technological University, Nanyang Avenue 639798, Singapore. ${ }^{5}$ Analysis and Test Center, Beijing University of Chemical Technology, Beijing 100029, China. ${ }^{6}$ Department of Chemistry, Tsinghua University, Beijing 100084, China. ${ }^{7}$ Department of Physics, Hong Kong Baptist University, Kowloon, Hong Kong SAR, China. ${ }^{凶}$ email: shiyantao@dlut.edu.cn; nitin_padture@brown.edu; yyzhou@hkbu.edu.hk
} 
$\mathrm{T}$ he past decade has witnessed the emergence of perovskite solar cells (PSCs) as a disruptive photovoltaic (PV) technology ${ }^{1-4}$. The state-of-the-art PSCs generally use organic-inorganic halide perovskites (OIHPs) with compositions based on formamidinium lead iodide $\left(\mathrm{FAPbI}_{3}\right)$ or methylammonium lead iodide $\left(\mathrm{MAPbI}_{3}\right)$, and they can deliver power conversion efficiencies (PCEs) up to $25.5 \%$, rivalling polycrystalline silicon solar cells $s^{5}$. The high PCEs are attributed to favorable optoelectronic properties of OIHPs, including high absorption coefficients, long carrier diffusion lengths, and high defect tolerance ${ }^{6-8}$. Also, OIHPs can be solution-processed at low temperatures, which enables the fabrication of lightweight and flexible $\mathrm{PSCs}^{9-12}$. Such combination of high performance and variable functionality makes PSCs appealing to numerous practical applications such as building-integrated PVs. Despite this promise, the deployment of PSCs has been held back due to concerns regarding their stability ${ }^{13}$. In this regard, there is a significant ongoing effort to make PSCs more stable while not compromising the device PCE.

Interface engineering is one of the most promising approaches for making efficient stable PSCs ${ }^{14-16}$. Conventional interfacial engineering entails either insertion of additional device layers (inorganic nanoparticles, polymers, molecules, etc.) or modification of surfaces using functional organic groups (thiophene, pyridine, etc.) and inorganic dopants (chlorine, alkali, etc. ${ }^{17-19}$. This is expected to improve the PCE and stability via optimizing energy-level alignment, improving interface contacts, suppressing structural defects, mitigating photocurrent hysteresis, or tailoring surface hydrophobicity ${ }^{14,17,20}$. But these interface-engineering methods may involve additional processing steps, possibly compromise the mechanical integrity of the interfaces in the resulting devices. Thus, it is critical to acquire device interfaces that are both functionally beneficial and mechanically robust for stable long-term device operation under continuous illumination as well as high endurance against repeated cyclic-bending (for flexible devices).

Herein we present a holistic design of an interpenetrating interface which is synthesized by deliberately reacting a predeposited FAI-incorporated $\mathrm{SnO}_{2}\left(\mathrm{FI}-\mathrm{SnO}_{2}\right)$ ETL layer and a $\mathrm{PbI}_{2}$-excess OIHP layer. Note that the $\mathrm{SnO}_{2}$-OIHP interface ${ }^{21-24}$ is chosen for a proof-of-concept demonstration for the new interface design. This fabrication approach is simple and potentially amenable to scalable processes compared with those similar interface structures previously reported ${ }^{25-27}$. Advanced characterizations, including time-of-flight secondary ion mass spectrometry (TOF-SIMS) and transmission electron microscopy (TEM), have been employed to confirm the interpenetrating structure. By probing the potential profile across the OIHP/ETL interpenetrating interface using Kelvin probe force microscopy (KPFM), we observed positive effects of the FI-SnO ${ }_{2}$ ETL on the heterojunction, electric field, and carrier dynamics. The champion PSC (rigid version) with this interface shows a high PCE of $22.2 \%$. After $1000 \mathrm{~h}$ continuous operation under one-sun intensity illumination, this device retains $82 \%$ of the initial PCE. We also demonstrate the use of this interface in flexible PSCs, which results in PCEs up to $20.1 \%$. The devices show remarkable mechanical endurance to repeated cyclic-bending fatigue, with a PCE retention of $85 \%$ after 2500 cycles, which is related to the enhanced structural integrity of this new interface as revealed by ex-situ cross-sectional scanning electron microscopy (SEM) characterization.

\section{Results}

Synthesis and characterization of the interpenetrating $\mathrm{SnO}_{2}$-OIHP interface. Figure 1a illustrates the preparation process for the FI-SnO ${ }_{2}$ ETL. A certain amount of FAI powder was first dissolved in an as-prepared $\mathrm{SnO}_{2}$-nanocrystals colloidal solution in isopropanol (IPA), which turns the solution from colorless to brownish color. Meanwhile, the FAI in the colloid has triggered the re-growth of $\mathrm{SnO}_{2}$ nanocrystals, probably caused by the hydrolysis reaction initiated by the $\mathrm{OH}^{-}$generated during the oxidation of $\mathrm{I}^{-}$(see Supplementary Fig. 1) ${ }^{28}$. Then, the solution was spin-coated on an FTO-coated glass substrate, followed by thermal annealing at $80^{\circ} \mathrm{C}$ for $2 \mathrm{~h}$ in the air and a sequential UV-ozone (UVO) treatment for $10 \mathrm{~min}$. The concentration of FAI in the $\mathrm{SnO}_{2}$ colloidal solution was optimized to $10 \mathrm{mg} \mathrm{mL}^{-1}$ based on the photovoltaic performance of the resulting PSCs (see Supplementary Fig. 2). The surface morphology of FI-SnO ${ }_{2}$ ETL was examined using scanning electron microscopy (SEM) and atomic force microscopy (AFM), and it appears to be very uniform, similar to that of pristine $\mathrm{SnO}_{2}$ ETL (see Supplementary Fig. 3). We further obtained conducting-AFM (C-AFM) maps for both FI-SnO ${ }_{2}$ and pristine $\mathrm{SnO}_{2}$ ETLs as shown in Fig. $1 \mathrm{~b}$ and c, respectively. While the electrical conductivity is uniform for both samples, more shunting locations are seen in the pristine $\mathrm{SnO}_{2}$ ETL, which can be attributed to the existence of more pinholes that allow direct contacts between the FTO and C-AFM tip ${ }^{28}$. This indicates that in the presence of $\mathrm{FAI}, \mathrm{SnO}_{2}$ nanocrystals are more uniformly distributed within the layer. We analyzed the composition of the FI-SnO $\mathrm{O}_{2}$ ETL. Figure 1d shows the Fouriertransform infrared spectroscopy (FTIR) spectrum of FI-SnO 2 together with the spectra from pristine $\mathrm{FAI}$ and $\mathrm{SnO}_{2}$ films. Two characteristic transmission bands at the wavenumbers of 1720 $\mathrm{cm}^{-1}$ and $1640 \mathrm{~cm}^{-1}$ are observed for FI-SnO ${ }_{2}$, which are assigned to the vibrations of the $\mathrm{C}=\mathrm{N}$ and the $\mathrm{N}-\mathrm{H}$ bonds, respectively, associated with $\mathrm{FA}^{+}$ions ${ }^{29}$. This suggests the presence of $\mathrm{FA}^{+}$ions in the ETL despite the thermal annealing and UVO treatments during the ETL preparation. X-ray photoelectron spectroscopy (XPS) spectra were also obtained for FI-SnO ${ }_{2}$ and pristine $\mathrm{SnO}_{2}$ films (Supplementary Figure 4). Besides N $1 s$ peak (at $400 \mathrm{eV}$ binding energy) for N-H, I $3 d$ peak (at $620 \mathrm{eV}$ binding energy) and $\mathrm{Cl} 2 p$ peak (at $199 \mathrm{eV}$ binding energy) are both very evident for $\mathrm{FI}-\mathrm{SnO}_{2}$, indicating the FAI has been partially converted to FACl. Importantly, as shown in Fig. 1e, the Sn $3 d$ doublet peaks for FI-SnO 2 shift to lower binding energies as compared to those for pristine $\mathrm{SnO}_{2}$. This is mostly attributed to the chemical interaction of Sn with less electronegative $\mathrm{I}$ and $\mathrm{Cl}$ in $\mathrm{FA}$ halide. All these results confirm that FI-SnO ${ }_{2}$ exhibits a nanocomposite thin film structure with $\mathrm{SnO}_{2}$ nanoparticles uniformly dispersed within an FA mixed halide (FAI and $\mathrm{FACl}$ ) matrix, as schematically illustrated in the right panel of Fig. 1a.

Once the FI-SnO ${ }_{2}$ ETL is prepared, we deposited the OIHP layer using a reported method ${ }^{30}$. Here, we used a precursor solution containing multi-ion compositions of $\mathrm{PbI}_{2}, \mathrm{PbBr}_{2}, \mathrm{CsI}$, FAI, and MABr (molar ratio: 1.15: 0.2: 0.05: 1.05: 0.2) in a mixed solvent of dimethylformamide (DMF) and dimethylsulfoxide (DMSO) (volume ratio: 4:1). The resulting OIHP thin film has a $\mathrm{Cs}_{0.04}\left(\mathrm{FA}_{0.84} \mathrm{MA}_{0.16}\right)_{0.96} \mathrm{~Pb}\left(\mathrm{I}_{0.84} \mathrm{Br}_{0.16}\right)_{3}$ composition with a slight excess of $\mathrm{PbI}_{2}$. The OIHP thin film was then annealed at $100^{\circ} \mathrm{C}$ for 50 min. During annealing, as shown schematically in Fig. 2a, the excess $\mathrm{PbI}_{2}$ in the OIHP thin film is expected to react with the FA halide in the FI-SnO ${ }_{2}$ ETL, resulting in a partial infiltration of the OIHP phase into FI-SnO ${ }_{2}$ ETL. Figure $2 \mathrm{~b}$ and c show cross-sectional SEM images of the as-deposited OIHP thin films on FI-SnO${ }_{2}$ and pristine $\mathrm{SnO}_{2}$ ETLs, respectively. Both OIHP thin films exhibit dense polycrystalline microstructures. We used time-of-flight secondary ion mass spectrometry (TOF-SIMS) to probe the through-thickness elemental distributions of $\mathrm{Pb}$ and $\mathrm{Sn}$ in the films. In order to exclude the influence of FTO (which contains Sn), OIHP/ETL films for TOF-SIMS 
a

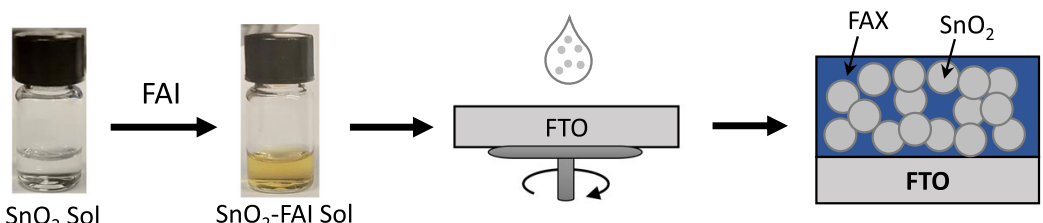

b

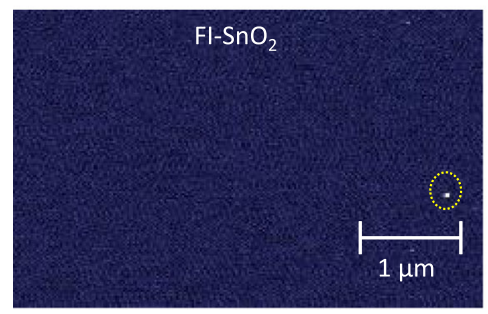

d

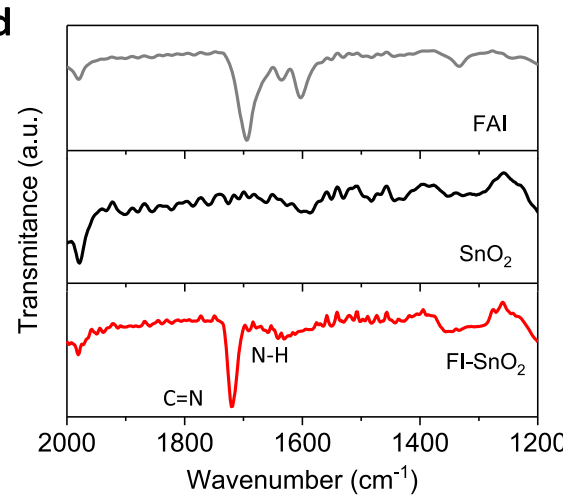

C

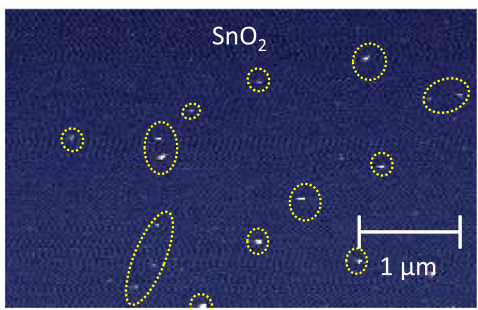

e

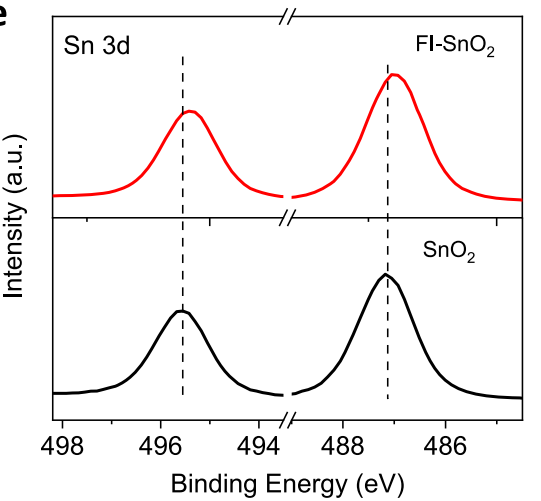

Fig. 1 ETL preparation and characterization. a Schematic illustration showing the synthesis process of FI-SnO $\mathrm{ETL}_{2} \mathbf{b}, \mathbf{c} \mathrm{C}-\mathrm{AFM}$ mapping of the FI-SnO 2 (left) and pristine $\mathrm{SnO}_{2}$ (right) ETLs on FTO substrates. d FTIR spectra of FAI, $\mathrm{SnO}_{2}$, and $\mathrm{FI}-\mathrm{SnO}_{2}$. e XPS spectra for $\mathrm{Sn} 3 d$ of FI-SnO 2 and pristine $\mathrm{SnO}_{2}$ ETLS.

analyses were deposited on bare glass substrates. Figure $2 \mathrm{~d}$ shows the depth profiles of $\mathrm{Pb}$ and $\mathrm{Sn}$ elemental concentrations revealed by TOF-SIMS (see the depth profiles of other elements in Supplementary Figure 5). With an increase in the sputtering time (corresponding to the depth into the film), for both films, the concentration of $\mathrm{Pb}$ (from OIHP) decreases whereas the $\mathrm{Sn}$ concentrations (from $\mathrm{SnO}_{2}$ ) increase. The vertical dashed line in Fig. 2d marks the beginning of the OIHP/ETL interface. As compared to the sample with the pristine $\mathrm{SnO}_{2} \mathrm{ETL}$, the $\mathrm{Pb}$ profile for the sample with the FI-SnO ${ }_{2}$ ETL exhibits a relatively shallower slope at the interface, which provides evidence for the interpenetration of the OIHP and the FI-SnO 2 ETL. To further prove this, we performed cross-sectional transmission electron microscope (TEM) characterization (Supplementary Fig. 6) and energy-dispersive X-ray spectroscopy (EDX) analysis (Fig. 2e, f) of the two OIHP/ETL films. The sample specimens were carefully prepared using focus ion beam (FIB) nanofabrication. Consistent with the cross-sectional SEM images, the thickness of the FI-SnO${ }_{2}$ film $(\sim 95 \mathrm{~nm})$ is approximately double that of the pristine $\mathrm{SnO}_{2}(\sim 45 \mathrm{~nm})$. $\mathrm{Pb}$ element, standing for OIHP, is evenly distributed in $\mathrm{FI}-\mathrm{SnO}_{2}$, clearly revealing the interpenetration of $\mathrm{SnO}_{2}$ and OIHP. For comparison, in the OIHP/pristine $\mathrm{SnO}_{2}$ structure, a relatively sharp transition in element distribution is shown between the ETL and OIHP, indicative of normal contact between the two layers without notable interpenetration. Considering all these observations, it is reasonable to deduce that an interfacial reaction has occurred between the excess $\mathrm{PbI}_{2}$ in $\mathrm{OIHP}$ and the FA halide in $\mathrm{FI}-\mathrm{SnO}_{2}$, leading to the formation of the OIHP phase within the FI-SnO ${ }_{2}$ ETL.
Electronic structure of the interpenetrating $\mathrm{SnO}_{2}-\mathrm{OIHP}$ interface. Based on the above characterization results, we confirm that the OIHP/FI-SnO ${ }_{2}$ interface comprises a mixed interlayer of $\mathrm{SnO}_{2}$ and OIHP phases, as illustrated in Fig. 2a. Ultraviolet photoemission spectroscopy (UPS) was used to determine the energy levels of those layers. As shown in Fig. 3a, the surface of the FI-SnO ${ }_{2}$ ETL and the pristine $\mathrm{SnO}_{2}$ ETL have similar Fermi levels of $3.8 \mathrm{eV}$ below the vacuum level, but the cutoff energy for FI-SnO ${ }_{2}$ ETL $(16.9 \mathrm{eV})$ is larger than that for pristine $\mathrm{SnO}_{2} \mathrm{ETL}$ $(16.7 \mathrm{eV})$. Considering the photon energy $(21.2 \mathrm{eV})$ of $\mathrm{He}-\mathrm{I}_{\alpha}$ radiation for the UPS measurements and the bandgap $(4.1 \mathrm{eV})$ of $\mathrm{SnO}_{2}$, the VBM levels are $-8.1 \mathrm{eV}$ and $-8.3 \mathrm{eV}$ and the CBM levels are $-4.2 \mathrm{eV}$ and $-4.0 \mathrm{eV}$, respectively, for $\mathrm{FI}-\mathrm{SnO}_{2}$ and pristine $\mathrm{SnO}_{2}$. Based on these results, the energy-level diagram for the OIHP/FI-SnO $\mathrm{S}_{2}$ interface is presented in Fig. 3c, which interestingly reveals a cascade electronic structure that is favorable for photocarriers transfer.

Furthermore, we employed in operando Kelvin probe force microscopy (KPFM) (Fig. 3d) to examine the potential profile along the cross-section in the device at the short-circuit condition under light illumination. KPFM has been proven to be a useful method for probe interface energetics in PSC devices ${ }^{31}$. Figure $3 \mathrm{e}, \mathrm{f}$ is KPFM images of the FI-SnO${ }_{2}$ and pristine $\mathrm{SnO}_{2}$ ETL-based devices, respectively, together with corresponding cross-sectional SEM images and contact potential difference (CPD) profiles. The related topological and phase atomic force microscopy (AFM) images are shown in Supplementary Fig. 7, demonstrating CPD results are independent of the surface topography 32,33 . The slope of the CPD profile suggests the existence of a $\mathrm{p}-\mathrm{n}$ junction. The slope degree 


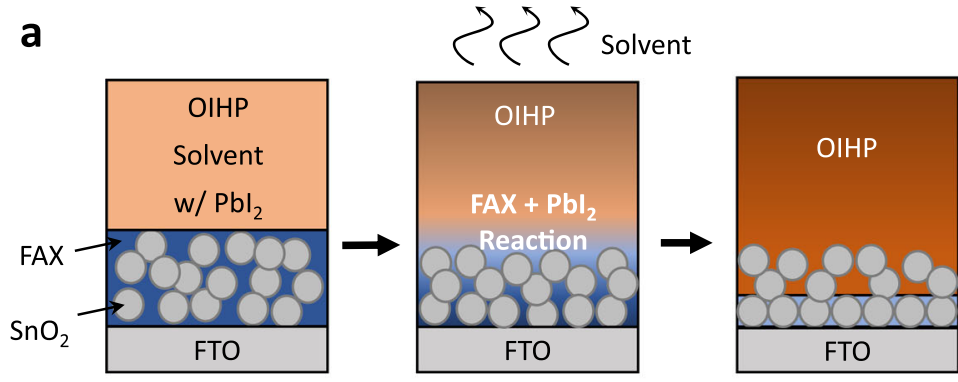

Annealing

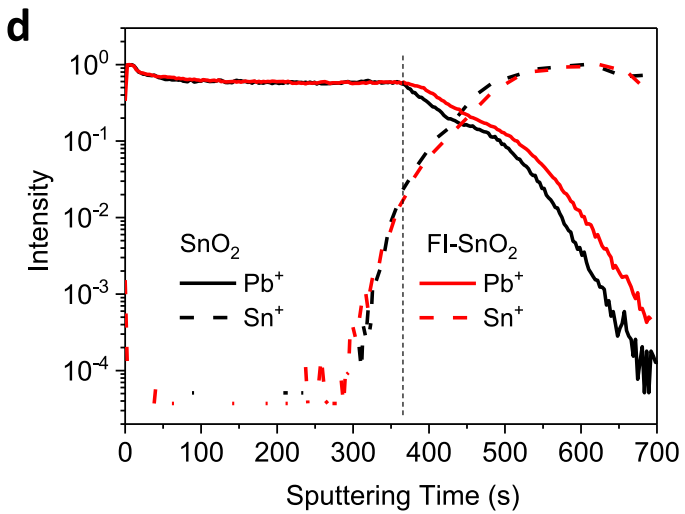

b

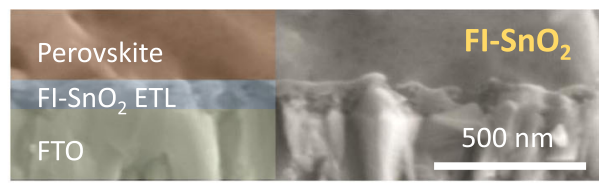

C

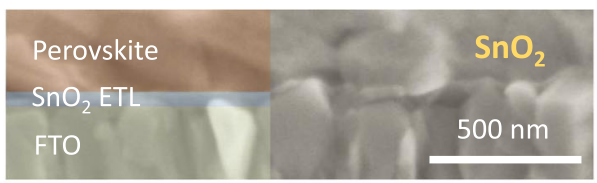

e
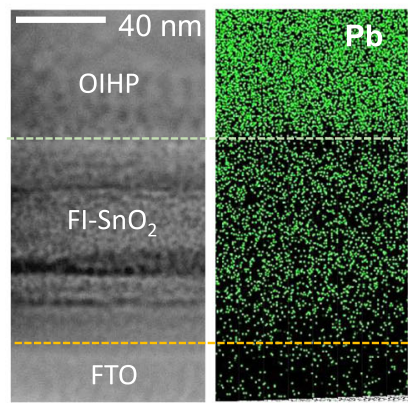

f
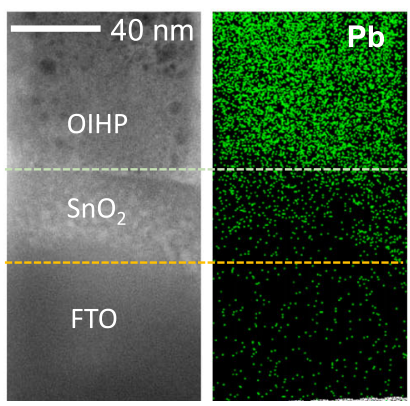

Fig. 2 Characterization of $\mathrm{SnO}_{\mathbf{2}}$-OIHP interfaces. a Schematic illustration showing the formation process of the $\mathrm{OlHP} / \mathrm{FI}-\mathrm{SnO} \mathrm{O}_{2}$ interface by the reaction between the excess $\mathrm{Pbl}_{2}$ in the OIHP layer and the FA halide in the FI-SnO $\mathrm{ST}_{2}$ ET. Cross-sectional SEM images of OIHP thin films on, $\mathbf{b}$ FI-SnO $\mathrm{ETL}_{2}$ and, $\mathbf{c}$ pristine $\mathrm{SnO}_{2}$ ETL. d TOF-SIMS depth elemental profiles of $\mathrm{Pb}$ and $\mathrm{Sn}$ into the OIHP/FI-SnO 2 and the OIHP/SnO ${ }_{2}$ films. Cross-sectional STEM image and EDX Pb-element mapping of OIHP films on, e $\mathrm{FI}-\mathrm{SnO}_{2} \mathrm{ETL}$ and, $\mathbf{f}$ pristine $\mathrm{SnO}_{2} \mathrm{ETL}$.

and range represent the driving force for exciton separation and the width of the depletion region, respectively. Compared with the small and narrow CPD drop at the OIHP/pristine $\mathrm{SnO}_{2}$ interface, the drop magnitude at the OIHP/FI-SnO ${ }_{2}$ interface is much larger $(0.1 \mathrm{~V}$ vs $0.03 \mathrm{~V})$, and the depletion region has penetrated into the OIHP layer. Based on the above results, we can deduce the photogenerated carrier transport behavior near the OIHP/ETL interface: as schematically illustrated in Fig. $3 g$, upon the device operation, the wider depletion region and larger potential difference near the OIHP/FI-SnO ${ }_{2}$ interface will greatly promote the separation of photogenerated carriers and the sequential collection of electrons. We then employed time-resolved photoluminescence (TRPL) and steady-state PL spectroscopy to study the carrier dynamics across the OIHP/ETL interface. The TRPL spectra in Fig. 3h show a faster biexponential PL decay for the OIHP/FI-SnO ETL, with lifetimes of $\tau_{1}=1.8 \mathrm{~ns}$ and $\tau_{2}=19.2 \mathrm{~ns}$, as compared to $\tau_{1}=2.3 \mathrm{~ns}$ and $\tau_{2}=20.5 \mathrm{~ns}$ for the OIHP/pristine $\mathrm{SnO}_{2}$ ETL. The shorter photocarrier recombination times is consistent with the more efficient PL quenching in the OIHP/FI-SnO 2 case, as revealed by the steady-state $\mathrm{PL}$ results in Fig. 3i. The enhanced charge dynamics of the OIHP/FI-SnO ${ }_{2}$ interface is attributed to its high structural and electronic integrity.

Device performance of perovskite solar cells in rigid and flexible versions. Rigid PSCs (on FTO-coated glass substrates) were fabricated after depositions of spiro-OMeTAD hole transporting layers (HTLs) and Au contacts on the as-formed OIHP/ ETL film structures. Figure 4a shows the cross-sectional SEM image of a typical PSC, where all the layers are labeled. The current density-voltage $(J-V)$ curves (forward and reverse scans) of the champion PSCs based on FI-SnO ${ }_{2}$ and pristine $\mathrm{SnO}_{2}$ ETLs are compared in Fig. 4b. The FI-SnO ${ }_{2}$-based PSC shows a high reversescan PCE of $22.2 \%$ with an open-circuit voltage $\left(V_{\mathrm{OC}}\right)$ of $1.18 \mathrm{~V}$, a fill factor $(\mathrm{FF})$ of 0.808 and a short-circuit current density $\left(J_{\mathrm{SC}}\right)$ of $23.2 \mathrm{~mA} \mathrm{~cm}^{-2}$, all higher than those for the pristine- $\mathrm{SnO}_{2}$-based PSC (PCE: 19.7\%; $V_{\mathrm{OC}}: 1.15 \mathrm{~V}$; FF:0.752; $J_{\mathrm{SC}}: 22.8 \mathrm{~mA} \mathrm{~cm}^{-2}$ ). The stabilized current/PCE outputs of the devices were further monitored at maximum-power-point (MPP) bias voltages, as shown in Fig. 4c. Stabilized PCEs of $22.1 \%$ and $18.8 \%$ are shown for PSCs based on the FI-SnO${ }_{2}$ and pristine $\mathrm{SnO}_{2}$ ETLs, respectively. The external quantum efficiency (EQE) spectra for both devices are shown in Fig. 4 d, where the integrated $J_{\mathrm{SC}}$ values are consistent with those extracted from the $J-V$ curves.

The carrier dynamics in both devices was further characterized by monitoring transient photovoltage decays. As shown in Fig. 4e, the $\mathrm{FI}-\mathrm{SnO}_{2}$-based device exhibits a carrier recombination lifetime $\tau_{r}(72 \mu \mathrm{s})$ that is much higher than that $(30 \mu \mathrm{s})$ of the pristine- $\mathrm{SnO}_{2}$-based device. This is in good agreement with the enhanced integrity of the OIHP/FI-SnO $\mathrm{Sn}_{2}$ interface and explains the higher device performance parameters. FI-SnO $\mathrm{STL}_{2}$ is prepared at low temperatures, and therefore, it is amenable to deposition on flexible polymer substrates for the fabrication of flexible PSCs. Figure $4 \mathrm{f}$ and Supplementary Fig. 8 show the $J-V$ curve (reverse scan) of the champion flexible PSC. Note that this device is made on a polyethylene naphthalate (PEN)/ITO substrate. A PCE of $20.1 \%$ is obtained under reverse $J-V$ scan direction, with $J_{S C}$ of 22.4 $\mathrm{mA} \mathrm{cm}{ }^{-2}, V_{\mathrm{OC}}$ of $1.15 \mathrm{~V}$, and $\mathrm{FF}$ of 0.782 . Under forward scan direction, the PCE is $19.6 \%$, with $J_{\mathrm{SC}}$ of $22.4 \mathrm{~mA} \mathrm{~cm}^{-2}, V_{\mathrm{OC}}$ of 1.14 $\mathrm{V}$, and FF of 0.769 . Upon MPP monitoring, a stabilized PCE of $19.8 \%$ is achieved. All these PV parameters are at the state-of-theart level for flexible PSCs ${ }^{34,35}$. 


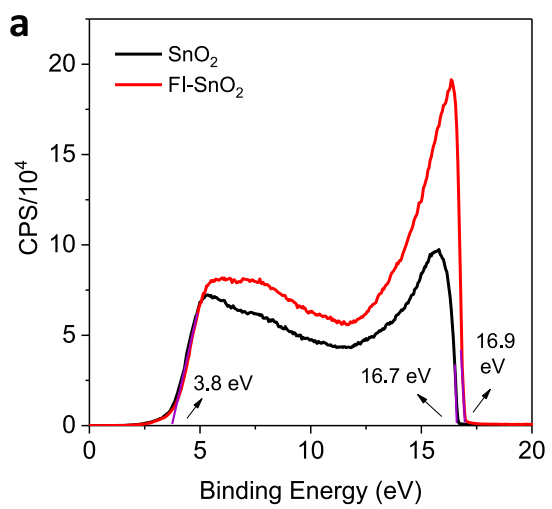

d

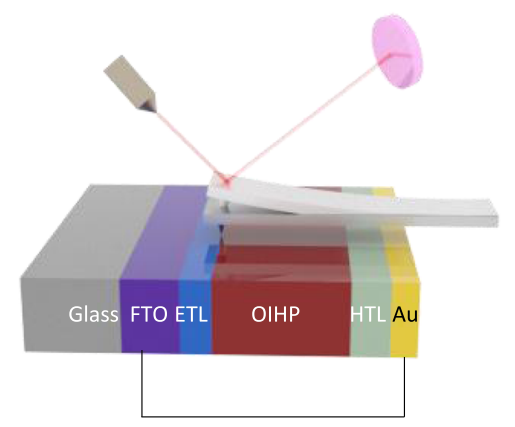

g

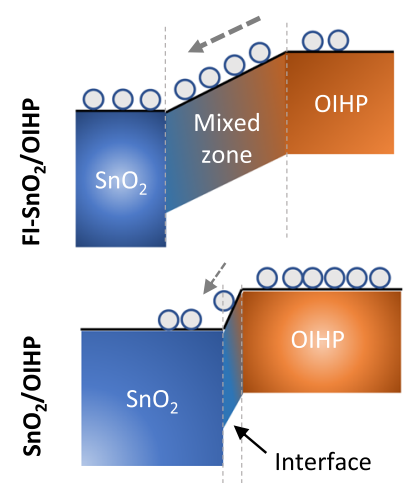

b

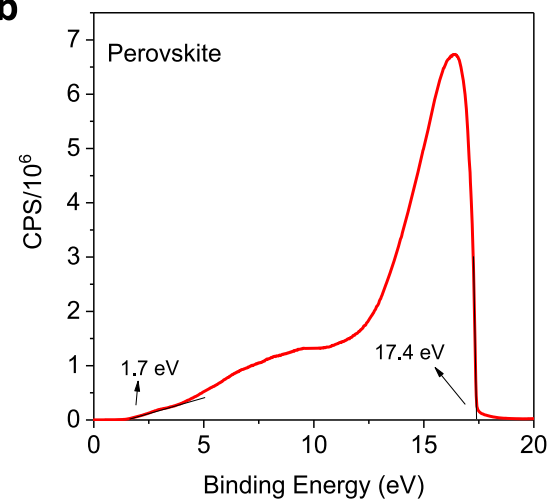

e

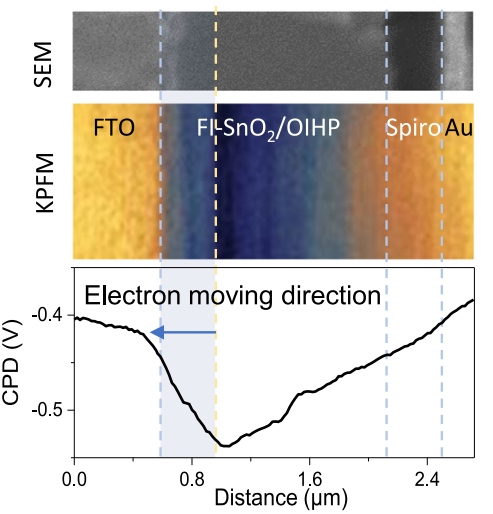

h

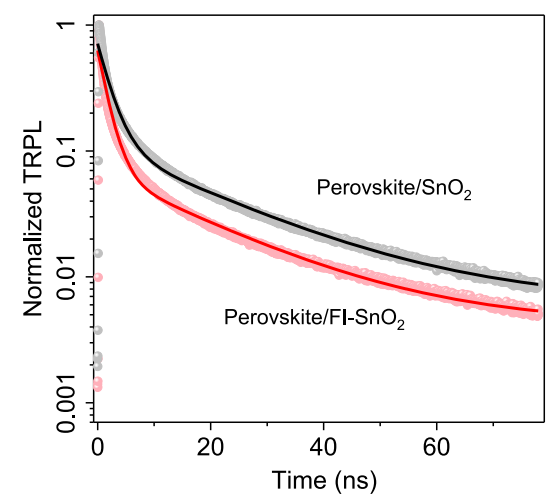

C

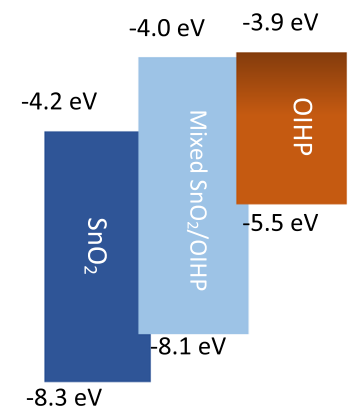

f

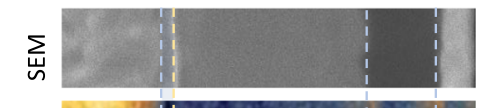

\begin{tabular}{ll}
\multicolumn{2}{c}{$m v$} \\
-462 & -367 \\
$\leftarrow$ & $\rightarrow$ \\
-590 & -564
\end{tabular}

FTO $\mathrm{SnO}_{2} / \mathrm{OIHP} \quad$ Spiro: $\mathrm{Au}$

$\sum_{\substack{\frac{1}{2} \\ \frac{0}{2}}}$

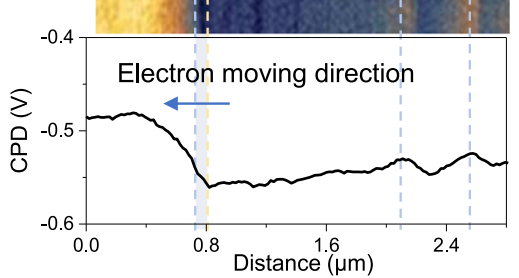

i

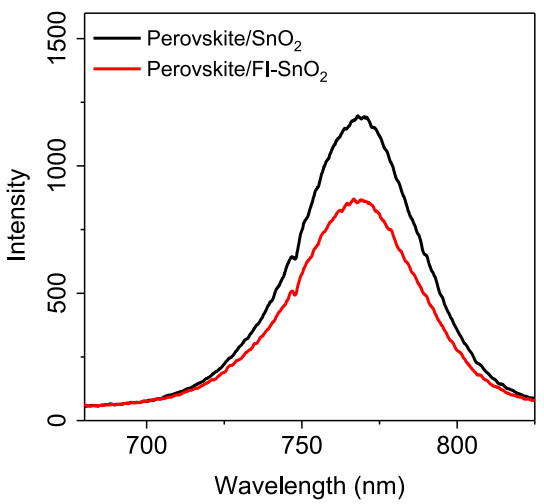

Fig. 3 Electronic properties of $\mathbf{S n O}_{\mathbf{2}}$-OIHP interfaces. UPS spectra showing the Fermi edge (left) and cutoff energy (right). a Fl-SnO ${ }_{2}$ and pristine $\mathrm{SnO} \mathrm{O}_{2}$ ETLs and $\mathbf{b}$ OIHP. c Energy-level diagram across the OIHP/FI-ETL interface. $\mathbf{d}$ Schematic illustration of cross-sectional KPFM measurement under shortcircuit condition. From top to bottom, SEM image, KPFM image, and contact potential difference (CPD; averaged) profile of the device on, e OIHP/FI-SnO 2 interface and, $\mathbf{f}$ pristine OIHP/ $\mathrm{SnO}_{2}$ interface. $\mathbf{g}$ Schematic illustration of electron transport characteristics near the $\mathrm{OlHP} / \mathrm{FI}-\mathrm{SnO}{ }_{2}$ and pristine OlHP/SnO${ }_{2}$ interfaces, respectively. $\mathbf{h}$ TRPL and $\mathbf{i}$ steady-state PL spectra of OIHP thin films on $\mathrm{FI}-\mathrm{SnO}_{2}$ and pristine $\mathrm{SnO}_{2} \mathrm{ETLs}$.

Operational stability and mechanical endurance of perovskite solar cells. The operational stability of rigid PSCs based on the FI- $\mathrm{SnO}_{2}$ and $\mathrm{SnO}_{2}$ ETLs is then compared. The initial PCEs are 21.1 and $19 \%$ for chosen PSCs based on $\mathrm{FI}-\mathrm{SnO}_{2}$ and pristine $\mathrm{SnO}_{2}$ ETLs, respectively. These devices were tested under MPP tracking with one-sun-intensity illumination. As shown in Fig. 5a, the PSC based on FI-SnO ${ }_{2}$ ETL clearly exhibits much slower PCE decay, and after the 1000 - $h$ continuous operation, $82 \%$ of the initial PCE was retained, demonstrating the long-term operational stability. For comparison, the PSC based on pristine $\mathrm{SnO}_{2}$ ETL shows a typical rapid PCE degradation during the initial period of the test $(72 \%$ retention after $400 \mathrm{~h})$. Such degradation has been attributed typically to the OIHP/ETL interface delamination ${ }^{36}$. In this context, our new interface design enhances the structural integrity of the OIHP/ETL interface, which leads to more operationally stable devices.

For flexible PSCs, repeated cyclic-bending fatigue tests were performed to evaluate the mechanical endurance. As illustrated in the inset of Fig. 5b, the minimum bending radius $(r)$ is $3 \mathrm{~mm}$, which corresponds to a maximum stress $(\sigma)$ of $371 \mathrm{MPa}$, calculated using the following relation ${ }^{37}$ :

$$
\sigma=\frac{E h}{2 r}
$$

where $E$ is the Young's modulus of the OIHP thin film and $h$ is the thickness of the substrate $(125 \mu \mathrm{m}$, neglecting other nanoscale layers in the device). Since the $E$ for the OIHP composition used here is not known, the $E$ value of $17.8 \mathrm{GPa}$ for $\mathrm{MAPbI}_{3} \mathrm{OIHP}$ is 
a

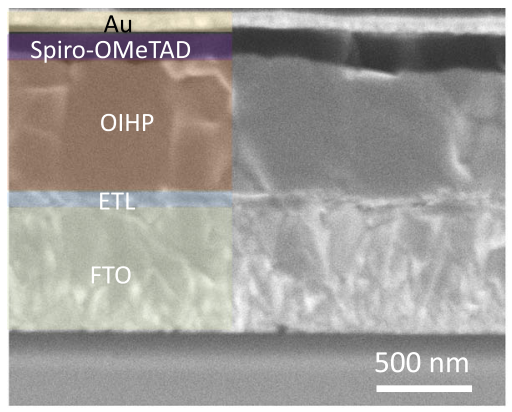

C

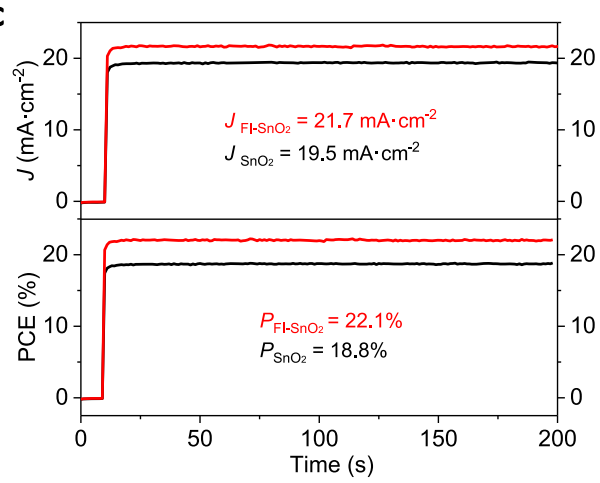

e

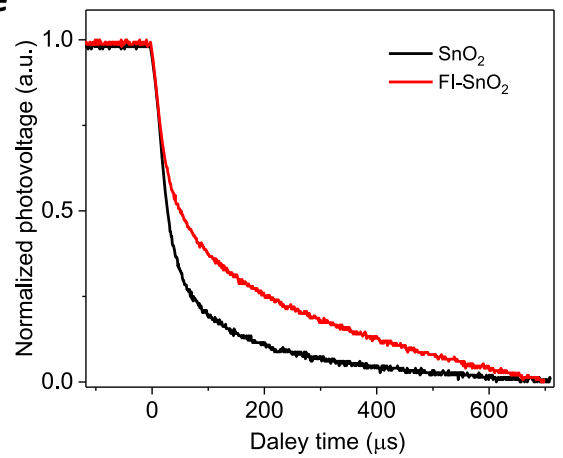

b

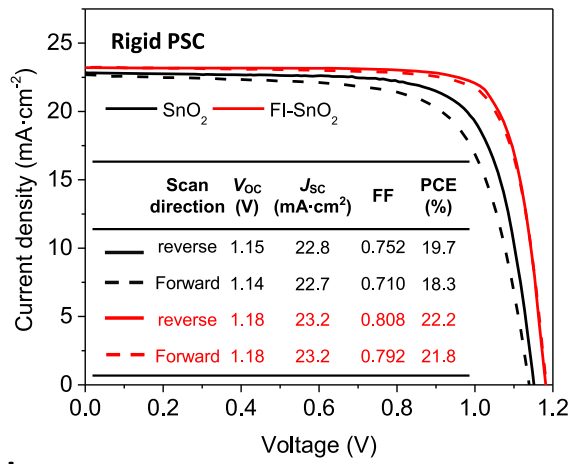

d

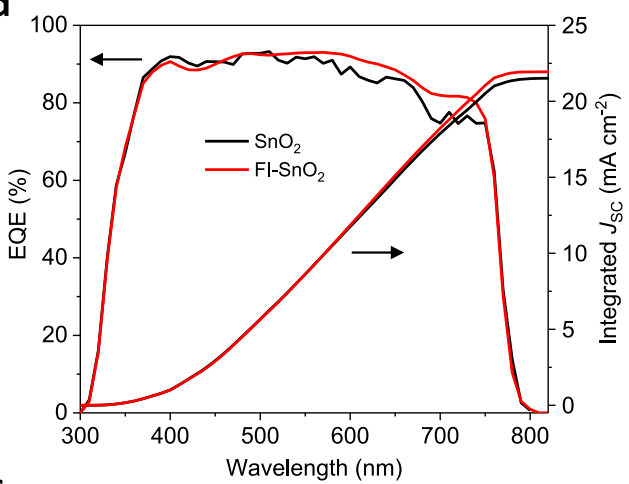

f

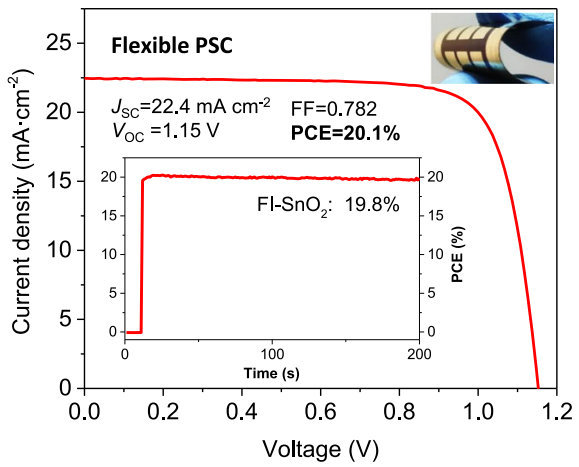

Fig. 4 Photovoltaic performance of rigid and flexible solar cell devices. a Cross-sectional SEM image of a rigid PSC device based on FI-SnO 2 ETL. b $J-V$ curves (forward and reverse scans) of rigid PSCs based on $\mathrm{FI}-\mathrm{SnO}_{2}$ and pristine $\mathrm{SnO}_{2}$ ETLs. The inset table shows the extracted PV parameters. c MPP current/PCE outputs, and $\mathbf{d}$ EQE spectra of the champion rigid PSCs based on $\mathrm{FI}-\mathrm{SnO}_{2}$ and pristine $\mathrm{SnO}_{2}$ ETLs. e Transient photovoltage decays for PSCs based on $\mathrm{FI}-\mathrm{SnO}_{2}$ and pristine $\mathrm{SnO}_{2}$ ETLs. $\mathbf{f} J-\mathrm{V}$ curve and MPP PCE output of the champion flexible PSCs based on FI-SnO $\mathrm{ETL}_{2}$. Inset is the photograph of a flexible PSC device.

used here, as the elastic properties of OIHPs are governed by the 3D framework of the lead-iodide octahedra ${ }^{37,38}$. In a full bending cycle, the OIHP thin film is subjected to the following stressing sequence: $0 \mathrm{MPa}$ (flat) to $+371 \mathrm{MPa}$ (convex; tension) to $0 \mathrm{MPa}$ (flat) to $-371 \mathrm{MPa}$ (concave; compression), and back to $0 \mathrm{MPa}$ (flat). As shown in Fig. $5 \mathrm{c}$, while in the concave state, the compressive strain will transform into a peel-off stress perpendicular to the OIHP/ETL interface. We resort to cross-sectional SEM images to determine the film and interface changes during bending cycles. As seen in Fig. 5d, e, after 1000 bending cycles, the OIHP film begins to peel off from the pristine $\mathrm{SnO}_{2}$ ETL, while the FI-SnO ${ }_{2}$ OIHP/ETL doesn't show obvious morphological change. When the bending cycle reaches 2500 , the pristine OIHP/SnO ${ }_{2}$ film shows a more obvious delamination phenomenon, and the $\mathrm{OIHP} / \mathrm{FI}-\mathrm{SnO}_{2}$ interface is still intact, except for some cracks appearing in the OIHP. Cracks on the OIHP caused by tensile stress will not so significantly hinder the longitudinal transport of photogenerated carriers as the delamination of OIHP from ETL substrate caused by compressive stress. Thus after 2500 such bending cycles, the PCE still retains $85 \%$ of the initial value for the FI-SnO ${ }_{2}$ ETL-based device, while only $60 \%$ is retained for the pristine- $\mathrm{SnO}_{2}$-ETL-based control device. This improved mechanical durability is attributed to the interpenetrating characteristics of the OHIP/ETL interface in the FI-SnO 2 ETL-based device. In fact, such diffuse interfaces are known to be more damage tolerant compared to sharp interfaces between two materials $^{39}$.

\section{Discussion}

We have developed an interpenetrating OIHP/ETL interface with enhanced structural integrity compared with its regular counterpart. We further characterized the interpenetrating characteristics of this interface using a set of advanced characterizations, well correlating the microstructure with the high performance and elucidating the underlying mechanisms. Incorporating this new interface enables the fabrication of efficient PSC devices that can deliver both long-term operational stability and high 
a

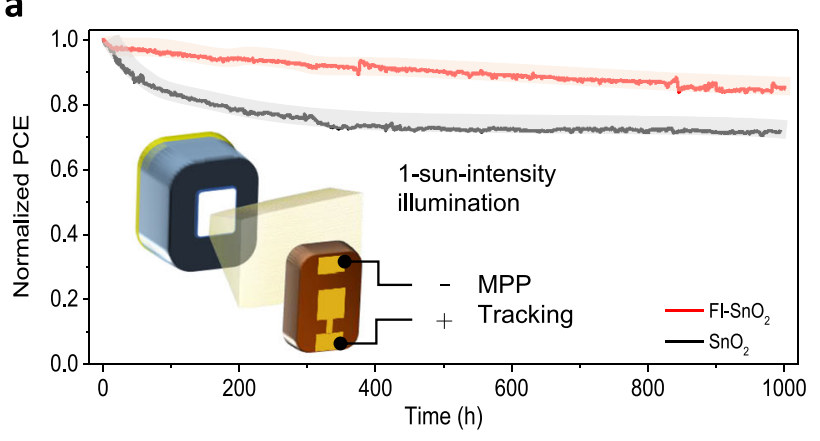

C

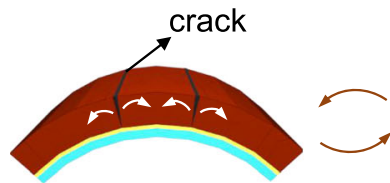

Tensile strain

d
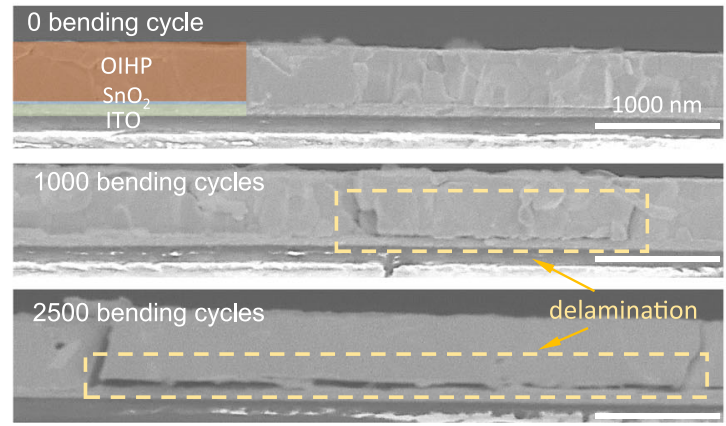

b

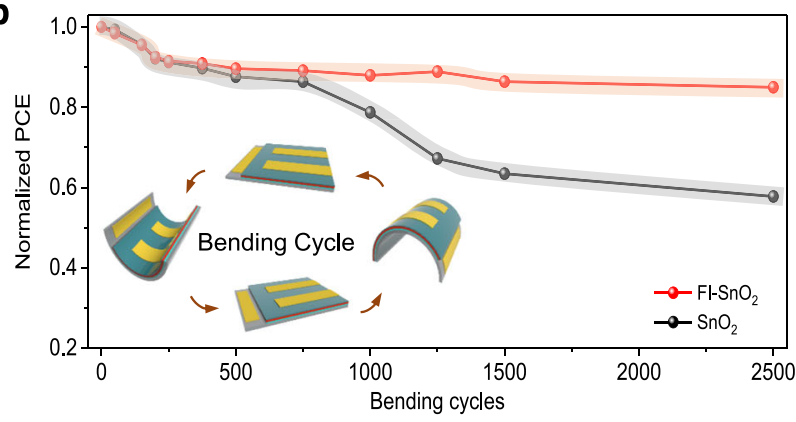

delamination

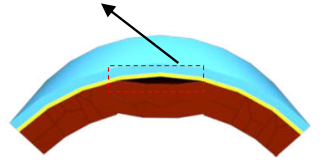

$\mathrm{SnO}_{2} / \mathrm{OIHP}$

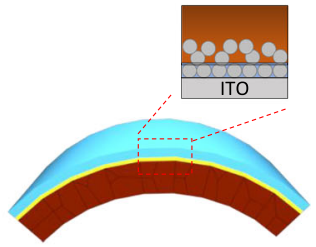

$\mathrm{FI}_{\mathrm{SnO}}$ /OIHP

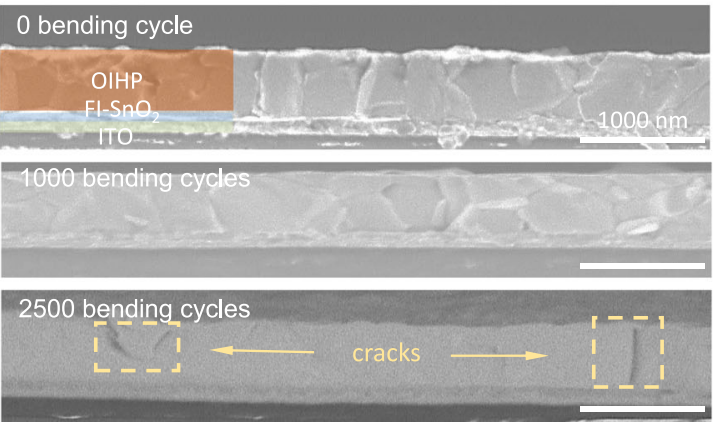

Fig. 5 Operational stability and mechanical durability of solar cell devices. a Operational stability of rigid $\mathrm{PSC}$ devices based on $\mathrm{Fl}-\mathrm{SnO} \mathrm{O}_{2}$ and pristine $\mathrm{SnO}_{2}$ ETLs under continuous light illumination (one-sun intensity illumination; flowing $\mathrm{N}_{2} ; 40{ }^{\circ} \mathrm{C}$ ). $\mathbf{b}$ Durability of flexible PSC devices based on $\mathrm{Fl}-\mathrm{SnO}{ }_{2}$ and pristine $\mathrm{SnO}_{2}$ ETLs as a function of mechanical bending cycles ( $40 \% \mathrm{RH}$; ambient air; $25^{\circ} \mathrm{C} ; 3 \mathrm{~mm}$ minimum r); Inset illustrates a typical mechanical bending cycle. c Schematic illustrations showing the strains in OIHP layers under different bending states (left panel) and the film states after bending cycles (right panel). d, e Cross-section SEM images of flexible PEN/ITO/SnO $/ 2 \mathrm{OIHP}$ film and PEN/ITO/FI-SnO $/ 2$ OIHP film after different bending cycles.

mechanical-fatigue endurance. This interface can not only retard ionic/molecular species to diffuse into the device, but also reduce the propensity for interfacial fracture, responsible for the device reliability enhancement. Furthermore, as identified by KPFM, the interpenetrating OIHP/ETL structure allows for more effective photocarriers separation and transport across the interface, compared to an abrupt OIHP/ETL interface, thus increasing the device PCE. While the case of OIHP/SnO 2 is demonstrated for proof-of-concept, this study points to a new interface-engineering strategy that can improve perovskite photovoltaics and electronics technologies for a variety of applications.

For future research, we are aware that there are more advanced characterization techniques currently being developed for understanding perovskites at micrometer to atomic scales, including synchrotron X-ray imaging, high-resolution scanning TEM, and tomographic AFM ${ }^{40-42}$. By using these techniques, we expect to reveal the detailed microstructure-property correlations in such interpenetrating interfaces. Also, it is worth gaining more insights into the interface formation mechanisms, which will open up new possibilities in the facile synthesis of more complex interfaces and precise tailoring of electronic properties and mechanical robustness for perovskite devices.

\section{Methods}

Raw chemicals. All the chemicals were used as received from commercial companies, including $\mathrm{PbI}_{2}$ and $\mathrm{PbBr}_{2}$ (>99\%, TCI, Japan), $\mathrm{CH}_{3} \mathrm{NH}_{3} \mathrm{Br}$ (MABr, 99.8\%,
Xi'an Polymer Light Technology Corp., China), $\mathrm{NH}_{2} \mathrm{CH}=\mathrm{NH}_{2} \mathrm{I}$ (FAI, 99.8\%, Xi'an Polymer Light Technology Corp., China), CsI (99.999\%, Alfa Aesar, UK), $\mathrm{SnCl}_{2} \cdot 2 \mathrm{H}_{2} \mathrm{O}$ (98-103\%, Alfa Aesar, UK), Spiro-OMeTAD (99.7\%, Lumtec Co., Taiwan), isopropanol (99.8\%, Sinopharm Chemical Reagent Co., Ltd, China). 4tert-butylpyridine (TBP, 96\%), Bis (trifluoromethane) sulfonamide lithium salt (99.95\%), and solvents acetonitrile (99.9\%), dimethylformamide (DMF, 99.8\%), dimethylsulfoxide (DMSO, 99.9\%), $\alpha, \alpha, \alpha$-Trifluorotoluene ( $\geq 99 \%)$ and chlorobenzene (CB, 99.8\%) were all purchased from Sigma Aldrich (USA). FTO-coated glass substrates $\left(7 \Omega \mathrm{sq}^{-1}\right)$ and PEN/ITO flexible substrates $\left(15 \Omega \mathrm{sq}^{-1}\right.$; $125-\mu \mathrm{m}$ thick) were purchased from Yingkou OPVtech New Energy Co. Ltd. (China).

Preparation of precursors and solutions. For the traditional ETL, $\mathrm{SnO}_{2}$ nanocrystal colloidal solution was prepared by dissolving $\mathrm{SnCl}_{2} \cdot 2 \mathrm{H}_{2} \mathrm{O}$ in anhydrous isopropanol $(0.1 \mathrm{M})$ in an open reflux apparatus and stirred at $85^{\circ} \mathrm{C}$ for $3 \mathrm{~h}$. After standing at $40^{\circ} \mathrm{C}$ for $3 \mathrm{~h}$, the sol was aged for over $24 \mathrm{~h}$ at room temperature. For the $\mathrm{FI}-\mathrm{SnO}_{2}$ ETL, the FAI powder has been dissolved in the above $\mathrm{SnO}_{2}$ sol with a concentration of $2 \mathrm{mg} \mathrm{mL}^{-1}, 10 \mathrm{mg} \mathrm{mL}^{-1}, 30 \mathrm{mg} \mathrm{mL}^{-1}$, and $60 \mathrm{mg} \mathrm{mL}^{-1}$. To prepare the $\mathrm{Cs}_{0.04}\left(\mathrm{FA}_{0.84} \mathrm{MA}_{0.16}\right)_{0.96} \mathrm{~Pb}\left(\mathrm{I}_{0.84} \mathrm{Br}_{0.16}\right)_{3}$ perovskite precursor, the mixed powder containing CsI (13.0 mg), FAI (180.6 mg), MABr (22.4 mg), $\mathrm{PbBr}_{2}(73.4$ $\mathrm{mg}$ ), and $\mathrm{PbI}_{2}(531.3 \mathrm{mg})$ were added into $1 \mathrm{~mL}$ mixed solvent of DMF and DMSO (volume ratio is 4:1), and stirring at $60^{\circ} \mathrm{C}$ for $30 \mathrm{~min}$. Precursor solution of HTL was prepared by dissolving $72.3 \mathrm{mg}$ spiro-MeOTAD, $28.8 \mu \mathrm{L}$ 4-tert-butylpyridine, $17.5 \mu \mathrm{L}$ lithium bis (trifluoromethylsulphonyl) imide acetonitrile solution $(520 \mathrm{mg}$ $\mathrm{mL}^{-1}$ ) into $1 \mathrm{~mL}$ chlorobenzene.

Device fabrication. Glass/FTO or the PEN/ITO substrates were etched with zinc powder and $4 \mathrm{M} \mathrm{HCl}$ to obtain the electrode pattern and then washed with cleaning fluid, deionized water, ethanol, and isopropanol sequentially. Subsequently, $\mathrm{SnO}_{2}$ sols were spin-coated on the substrates at $2000 \mathrm{rpm}$ for $30 \mathrm{~s}$, and then heated at $80^{\circ} \mathrm{C}$ for $2 \mathrm{~h}$ to remove the solvent. All ETLs were then UVO-posttreated for $10 \mathrm{~min}$. Finally, the ETL substrates were transferred to the glove box 
$\left(\mathrm{H}_{2} \mathrm{O}<0.01 \mathrm{ppm}, \mathrm{O}_{2}<0.01 \mathrm{ppm}\right)$. To prepare the perovskite films, $50 \mu \mathrm{L}$ above perovskite precursor was spread on the $\mathrm{SnO}_{2}$-ETL substrates, followed by a twostage spin-coating process (1000 rpm for $10 \mathrm{~s}$ and $6000 \mathrm{rpm}$ for $30 \mathrm{~s}$ ). During the second spin-coating stage, $250 \mu \mathrm{L}$ of $\alpha, \alpha$, $\alpha$-trifluorotoluene was dripped on the spinning substrate $15 \mathrm{~s}$ prior to the end of the program. The substrates were then annealed at $100^{\circ} \mathrm{C}$ for $50 \mathrm{~min}$. Subsequently, HTL was deposited on top of the perovskite layer by spin coating at $3000 \mathrm{rpm}$ for $30 \mathrm{~s}$. Finally, a $60-\mathrm{nm}$ Au electrode with an active area of $0.16 \mathrm{~cm}^{2}$ was thermally evaporated on top of the HTL.

Materials characterization. The microstructures of the thin films were observed using field-emission SEM instruments (JSM-7401F, JEOL, Japan; Merlin, Zeiss, Germany). To obtain the cross-sectional SEM images, the flexible samples were embrittled with liquid nitrogen in the glove box $\left(\mathrm{H}_{2} \mathrm{O}<0.01 \mathrm{ppm}, \mathrm{O}_{2}<0.01 \mathrm{ppm}\right)$ and fractured with two tweezers. The AFM, C-AFM images for ETL surfaces were obtained using an atomic force microscope (Dimension Icon, BRUKER, USA) in the contact mode. A multi-75E-G probe (PF TUNA) was used for the C-AFM detection. To obtain the samples for cross-sectional KPFM measurements, the devices were cleaved near the active area of the solar cells using a diamond cutter and then fractured by tension stress to expose the cross-section. The KPFM images of PSCs were obtained using the same model Bruker AFM as C-AFM measurement, while a conductive Antimony (n) doped Si Rtespa-300 probe (BRUKER, USA) with the frequency of $300 \mathrm{KHz}$ and spring constants of $40 \mathrm{~N} \mathrm{~m}^{-1}$ was used for the detection. A standard AC mode, at a scanning rate of $1 \mathrm{~Hz}, \mathrm{AC}$ voltage of $0.5 \mathrm{~V}$, and frequency of $73 \mathrm{kHz}$, was used for amplitude modulation (AM-KPFM) measurement. The cross-sectional STEM specimen were prepared using a Focused Ion Beam nanofabrication platform (FEI Nanolab 600i, Thermo Fisher, USA). To protect the surface from damage during FIB milling, the cross-sectional surface was in situ-coated with platinum using an FEI gas injection system. Then the STEM images and EDS mappings were obtained on an aberration-corrected electron microscope (ARM200F, JEOL, Japan) at an acceleration voltage of $200 \mathrm{kV}$ and beam convergence angle of $27 \mathrm{mrad}$. The collection angles were set to $68-280$ and $45 \mathrm{mrad}$ for HAADF and BF imaging, respectively. The TEM images of $\mathrm{SnO}_{2}$ nanocrystallines (scraped from the substrates) were performed on $2100 \mathrm{~F}$ (JEOL) instrument using an acceleration voltage of $200 \mathrm{KV}$. Steady-state and time-resolved PL spectra were recorded using a spectrophotometer (Varian Cary Eclipse Fluorescence, Agilent, USA) operated at $395 \mathrm{~nm}$ excitation. FTIR was obtained in attenuated total reflection (ATR) mode using an infrared spectrometer (V70, Bruker, USA). XPS was performed using Kratos Analytical spectrometer (AXIS ULTRA HAS, Kratos Analytical, UK) and all XPS spectra were shifted to account for sample charging using inorganic carbon at $284.80 \mathrm{eV}$ as a reference. UPS was performed using Kratos Analytical spectrometer (AXIS ULTRA DLD, Kratos Analytical, UK), and mono-chromatized $\mathrm{He}-\mathrm{I}_{\alpha}$ radiation at $21.2 \mathrm{eV}$ was used. The ToF-SIMS measurements (Model TOF-SIMS 5, ION-TOF GmbH, Germany) were performed with the pulsed primary ions from a GICB $(10 \mathrm{keV})$ liquid-metal ion gun for the sputtering and $\mathrm{a}_{3}{ }^{++}$pulsed primary ion beam for the analysis $(60$ $\mathrm{keV})$. The noninterlaced mode was used, with $1 \mathrm{~s}$ of sputtering followed by $3 \mathrm{~s}$ of analysis. The analysis area was $100 \times 100 \mu \mathrm{m}$, and the sputter rate was calibrated with the $\mathrm{SiO}_{2}$ substrate on each batch of samples.

Solar cell performance testing. The current density-voltage $(J-V)$ characteristics of PSCs were measured by a Source Meter (2400, Keithley, USA) at the scan speed of $100 \mathrm{mV} \mathrm{s}^{-1}$ under AM $1.5 \mathrm{G}$ one-sun illumination $\left(100 \mathrm{~mW} \mathrm{~cm}^{-2}\right)$ generated by a solar simulator (Oriel Sol3A Class AAA, Newport, USA) in air. The intensity was calibrated using a VLSI standard incorporated PN 91150V-KG3 Si reference cell. The active device area of PSCs during the measurements is $0.096 \mathrm{~cm}^{2}$ defined by a metal mask with a $0.35-\mathrm{cm}$ diameter circular hole. Steady-state current/PCE outputs were measured using 2400 Source Meter (Keithley, USA) at voltages determined from the MPPs of the reverse-scan J-V curves. The EQE spectra were obtained using a quantum efficiency measurement system (Oriel IQE 200B, Newport, USA).

Solar cell stability testing. For long-term operational stability tests, unencapsulated PSCs were placed in a sealed cell holder with a transparent quartz cover. A continuous flow of $\mathrm{N}_{2}$ gas was passed through the holder to minimize the water and oxygen content in the atmosphere. The PSCs were biased at the maximumpower-point voltage using a potentiostat under continuous one-sun-intensity white-LED illumination at around $45^{\circ} \mathrm{C}$. For mechanical bending tests, the ben radius is $3 \mathrm{~mm}$. Each bending cycle consists of the device geometry status change of flat $\rightarrow$ convex $\rightarrow$ flat $\rightarrow$ concave $\rightarrow$ flat (as schematically illustrated in the inset of Fig. $5 b) . J-V$ curves were measured under the ambient condition to monitor the device performance decay.

Reporting summary. Further information on research design is available in the Nature Research Reporting Summary linked to this article.

\section{Data availability}

The data that support the findings of this study are available from the corresponding authors upon reasonable request.
Received: 8 June 2020; Accepted: 14 January 2021;

Published online: 12 February 2021

\section{References}

1. Burschka, J. et al. Sequential deposition as a route to high-performance perovskite-sensitized solar cells. Nature 499, 316-319 (2013).

2. Jeon, N. J. et al. Compositional engineering of perovskite materials for highperformance solar cells. Nature 517, 476 (2015).

3. Jiang, Q. et al. Surface passivation of perovskite film for efficient solar cells. Nat. Photonics 13, 460-466 (2019).

4. Dunlap-Shohl, W. A., Zhou, Y., Padture, N. P. \& Mitzi, D. B. Synthetic approaches for halide perovskite thin films. Chem. Rev. 119, 3193-3295 (2019).

5. NREL. Best research-cell efficiencies. https://www.nrel.gov/pv/cell-efficiency. html (2020).

6. Eperon, G. E. et al. Formamidinium lead trihalide: a broadly tunable perovskite for efficient planar heterojunction solar cells. Energy Environ. Sci. 7, 982-988 (2014).

7. Dong, Q. et al. Electron-hole diffusion lengths $>175 \mu \mathrm{m}$ in solution-grown $\mathrm{CH}_{3} \mathrm{NH}_{3} \mathrm{PbI}_{3}$ single crystals. Science 347, 967-970 (2015).

8. Kovalenko, M. V., Protesescu, L. \& Bodnarchuk, M. I. Properties and potential optoelectronic applications of lead halide perovskite nanocrystals. Science 358, 745-750 (2017).

9. Wang, C. et al. Water vapor treatment of low-temperature deposited $\mathrm{SnO}_{2}$ electron selective layers for efficient flexible perovskite solar cells. ACS Energy Lett. 2, 2118-2124 (2017).

10. Di Giacomo, F., Fakharuddin, A., Jose, R. \& Brown, T. M. Progress, challenges and perspectives in flexible perovskite solar cells. Energy Environ. Sci. 9, 3007-3035 (2016).

11. Yang, D., Yang, R., Priya, S. \& Liu, S. Recent advances in flexible perovskite solar cells: fabrication and applications. Angew. Chem. Int Ed. 58, 4466-4483 (2018).

12. Huang, W. et al. Efficient and mechanically robust ultraflexible organic solar cells based on mixed acceptors. Joule 4, 128-141 (2020).

13. Niu, G., Guo, X. \& Wang, L. Review of recent progress in chemical stability of perovskite solar cells. J. Mater. Chem. A 3, 8970-8980 (2015).

14. Rajagopal, A., Yao, K. \& Jen, A. K.-Y. Toward perovskite solar cell commercialization: a perspective and research roadmap based on interfacial engineering. Adv. Mater. 30, 1800455 (2018).

15. Bai, Y., Meng, X. \& Yang, S. Interface engineering for highly efficient and stable planar p-i-n perovskite solar cells. Adv. Energy Mater. 8, 1701883 (2018).

16. $\mathrm{Li}, \mathrm{W}$. et al. Enhanced UV-light stability of planar heterojunction perovskite solar cells with caesium bromide interface modification. Energy Environ. Sci. 9, 490-498 (2016).

17. Cho, A.-N. \& Park, N.-G. Impact of interfacial layers in perovskite solar cells. ChemSusChem 10, 3687-3704 (2017).

18. Tan, H. et al. Efficient and stable solution-processed planar perovskite solar cells via contact passivation. Science 355, 722-726 (2017).

19. Zhu, P. et al. Simultaneous contact and grain-boundary passivation in planar perovskite solar cells using $\mathrm{SnO} 2-\mathrm{KCl}$ composite electron transport layer. $A d v$. Energy Mater. 10, 1903083 (2020).

20. Yang, S. et al. Functionalization of perovskite thin films with moisture-tolerant molecules. Nat. Energy 1, 15016 (2016).

21. Ke, W. et al. Low-temperature solution-processed tin oxide as an alternative electron transporting layer for efficient perovskite solar cells. J. Am. Chem. Soc. 137, 6730-6733 (2015).

22. Jiang, Q. et al. Enhanced electron extraction using $\mathrm{SnO}_{2}$ for high-efficiency planar-structure $\mathrm{HC}(\mathrm{NH} 2) 2 \mathrm{PbI} 3-$ based perovskite solar cells. Nat. Energy 2, 16177 (2016)

23. Li, Z. et al. Spontaneous interface ion exchange: passivating surface defects of perovskite solar cells with enhanced photovoltage. Adv. Energy Mater. 9, 1902142 (2019).

24. Guo, Z. et al. VOC over $1.4 \mathrm{~V}$ for amorphous tin-oxide-based dopant-free CsPbI2Br perovskite solar cells. J. Am. Chem. Soc. 142, 9725-9734 (2020).

25. Hou, Y. et al. A band-edge potential gradient heterostructure to enhance electron extraction efficiency of the electron transport layer in highperformance perovskite solar cells. Adv. Funct. Mater. 27, 1700878 (2017).

26. Ke, W. et al. Cooperative tin oxide fullerene electron selective layers for highperformance planar perovskite solar cells. J. Mater. Chem. A 4, 14276-14283 (2016).

27. $\mathrm{Wu}, \mathrm{Y}$. et al. Perovskite solar cells with $18.21 \%$ efficiency and area over $1 \mathrm{~cm}^{2}$ fabricated by heterojunction engineering. Nat. Energy 1, 16148 (2016).

28. Dong, Q. et al. Improved $\mathrm{SnO}_{2}$ electron transport layers solution-deposited at near room temperature for rigid or flexible perovskite solar cells with high efficiencies. Adv. Energy Mater. 9, 1900834 (2019). 
29. Dou, B. et al. Degradation of highly alloyed metal halide perovskite precursor inks: mechanism and storage solutions. ACS Energy Lett. 3, 979-985 (2018).

30. Anaraki, E. H. et al. Highly efficient and stable planar perovskite solar cells by solution-processed tin oxide. Energy Environ. Sci. 9, 3128-3134 (2016).

31. Weber, S. A. L. et al. How the formation of interfacial charge causes hysteresis in perovskite solar cells. Energy Environ. Sci. 11, 2404-2413 (2018).

32. Byeon, J. et al. Charge transport layer-dependent electronic band bending in perovskite solar cells and its correlation to light-induced device degradation. ACS Energy Lett. 5, 2580-2589 (2020).

33. Cui, P. et al. Planar $\mathrm{p}-\mathrm{n}$ homojunction perovskite solar cells with efficiency exceeding 21.3\%. Nat. Energy 4, 150-159 (2019).

34. Huang, K. et al. High-performance flexible perovskite solar cells via precise control of electron transport layer. Adv. Energy Mater. 9, 1901419 (2019).

35. Jung, H. S., Han, G. S., Park, N.-G. \& Ko, M. J. Flexible perovskite solar cells. Joule 3, 1850-1880 (2019).

36. Soufiani, A. M. et al. Lessons learnt from spatially resolved electro- and photoluminescence imaging: Interfacial delamination in $\mathrm{CH}_{3} \mathrm{NH}_{3} \mathrm{PbI}_{3}$ planar perovskite solar cells upon illumination. Adv. Energy Mater. 7, 1602111 (2017).

37. Yadavalli, S. K., Dai, Z., Zhou, H., Zhou, Y. \& Padture, N. P. Facile healing of cracks in organic-inorganic halide perovskite thin films. Acta Mater. 187, 112-121 (2020).

38. Ramirez, C., Yadavalli, S. K., Garces, H. F., Zhou, Y. \& Padture, N. P. Thermomechanical behavior of organic-inorganic halide perovskites for solar cells. Scr. Mater. 150, 36-41 (2018).

39. Suresh, S. \& Mortensen, A. Fundamentals of Functionally Graded Materials: Processing and Thermomechanical Behavior of Graded Metals and Metalceramic Composites (IOM Communications Ltd., 1998).

40. Cai, S. \& Zhou, Y. Visualizing the invisible in perovskites. Joule 4, 2545-2548 (2020).

41. Zhou, Y., Zhou, H., Deng, J., Cha, W. \& Cai, Z. Decisive structural and functional characterization of halide perovskites with synchrotron. Matter 2, 360-377 (2020).

42. Song, J., Zhou, Y., Padture, N. P. \& Huey, B. D. Anomalous 3D nanoscale photoconduction in hybrid perovskite semiconductors revealed by tomographic atomic force microscopy. Nat. Commun. 11, 3308 (2020).

\section{Acknowledgements}

The work at Brown University was funded by the National Science Foundation (Grant No. OIA-1538893). The work at the Dalian University of Technology was financially supported by the National Natural Science Foundation of China (Grant Nos. 51773025, 22005043, and 51872036), Natural Science Foundation of Liaoning Province, China (Grant No. 20180510027), Dalian Science and Technology Innovation Fund (Grant No. 2019J12GX032), and China Postdoctoral Science Foundation (Grant Nos. 2019TQ0046 and 2020M680941). Experimental assistance from Mr. T. Shen, Dr. B. Zhao, Mr. J. Zhang, Dr. X. Jiang, and Prof. X. Song is also acknowledged.

\section{Author contributions}

Y.Z. and N.P.P. conceived the idea and supervised the project. Y.Z. and Q.D. designed the experiment. Q.D. performed the perovskite synthesis, most of the characterizations (SEM, UV-vis, FTIR, TOF-SIMS, XPS, UPS, etc.), and device fabrication/testing. C.Z., X.C., Y.H. and Z.L. performed the TEM characterization and the FIB specimen preparation. M.C. assisted in the PL, AFM, and KPFM measurements and analysis. C.J., Y.F., and Y.L. assisted in the flexible device fabrication and testing. J.G. assisted in the schematic drawing. M.C., Z.D. and M.H. performed the MPP stability measurements. S.K.Y. contributed to the discussion on mechanical bending tests. Y.S. and L.W. contributed to the discussion on the material and device characteristics. Q.D., Y.S., Y.Z. and N.P.P prepared the paper with contributions from all the other co-authors.

\section{Competing interests}

The authors declare no competing interests.

\section{Additional information}

Supplementary information The online version contains supplementary material available at https://doi.org/10.1038/s41467-021-21292-3.

Correspondence and requests for materials should be addressed to Y.S., N.P.P. or Y.Z

Peer review information Nature Communications thanks Tsutomu Miyasaka and the other, anonymous, reviewer(s) for their contribution to the peer review of this work.

Reprints and permission information is available at http://www.nature.com/reprints

Publisher's note Springer Nature remains neutral with regard to jurisdictional claims in published maps and institutional affiliations.

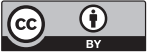

Open Access This article is licensed under a Creative Commons Attribution 4.0 International License, which permits use, sharing, adaptation, distribution and reproduction in any medium or format, as long as you give appropriate credit to the original author(s) and the source, provide a link to the Creative Commons license, and indicate if changes were made. The images or other third party material in this article are included in the article's Creative Commons license, unless indicated otherwise in a credit line to the material. If material is not included in the article's Creative Commons license and your intended use is not permitted by statutory regulation or exceeds the permitted use, you will need to obtain permission directly from the copyright holder. To view a copy of this license, visit http://creativecommons.org/ licenses/by/4.0/

(C) The Author(s) 2021 